\title{
INFLUENCIA DE LOS CORREDORES ECONÓMICOS EN LA PRODUCCIÓN DE LIMÓN DE EXPORTACIÓN EN LA REGIÓN PIURA*
}

\author{
Mercedes Soto Plasencia \\ UNIVERSIDAD DE LIMA \\ SOTANA20@aloe.ulima.edu.pe
}

\begin{abstract}
Resumen
Este artículo aplica enfoques y conceptos vinculados a los modelos de ventajas competitivas y ventajas comparativas, y de corredores económicos -cadenas productivas, clusters y conglomerados- al análisis de las posibilidades de mejoramiento de la producción del limón en el norte del país, con miras a incorporar este fruto a la oferta exportable hacia los mercados del Asia Pacífico. Con este fin, en la primera parte se discute la relevancia de adoptar una estrategia basada en el desarrollo de corredores económicos como alternativa eficiente para combinar factores de escala de producción con eficiencia de costos logísticos, específicamente en los corredores de Sullana-Tambogrande-San Lorenzo-Ayabaca y de Chulucanas-Morropón-Huancabamba En la segunda parte se adopta una visión sistémica de la competitividad agroindustrial que permite identificar las barreras que limitan y las condiciones ex ante que afectan positivamente la producción de limón: tecnificación, asociatividad de los agricultores, acceso a financiación, adopción de métodos mecanizados de siembra-cosecha, entre otros.
\end{abstract}

Palabras clave: productividad y calidad de la producción exportable de limón, productos agroindustriales de la región Piura, corredores económicos, cadena productiva del limón.

\begin{abstract}
This paper uses the approaches and concepts of competitive and comparative advantage models and the principles of economic corridors, including production chains, clusters and conglomerates, to analyze the potential for improved lime production in Peru's northern regions for export to Asia Pacific markets. The first section discusses the relevance of adopting a strategy based on economic corridors as an efficient alternative to combine scale of production and efficient logistics costs, in particular for the Sullana-Tambogrande-San Lorenzo-Ayabaca and ChulucanasMorropon-Huancabamba corridors. The second section adopts a systemic view of agro-industrial competitiveness to identify the barriers to and ex ante conditions that have a positive impact on lime production, namely adoption of technologies, growers' associations, access to financing, adoption of mechanized planting and harvesting techniques, and others.
\end{abstract}

Key words: productivity and quality of lime exports, Piura region agro-industrial exports, economic corridors, lime production chain.

* Trabajo premiado en el II Concurso de Investigación Juan Abugattás Abugattás, convocado en el 2005 por la Red Peruana para Estudios del Asia Pacífico (Redap). 


\section{Introducción}

En la región Piura se encuentran dos espacios marcadamente diferenciados: la costa y la sierra. La costa ha sido privilegiada por el Estado a través de importantes inversiones en diversos sectores: hidrocarburos (petróleo), agrícola (proyecto Chira-Piura), pesquero (terminales pesqueros), transportes (carreteras asfaltadas, puertos y aeropuertos) y educación (universidad e institutos tecnológicos y pedagógicos). La sierra, por el contrario, no ha recibido el impulso de las grandes inversiones gubernamentales: carece de infraestructura básica, la calidad de sus recursos naturales es baja, sus recursos humanos no son calificados; todo lo cual genera altos costos de transacción.

La sierra piurana constituye un espacio desarticulado con predominancia de la actividad agropecuaria que, en su mayor parte, es una agricultura de subsistencia. Se producen bienes con escaso valor agregado, y los pocos productos que ganan reconocimiento sostenido en el mercado externo, como mango, espárrago, limón y café, se exportan casi en su forma natural, dejando que los procesos de envasado, etiquetado y comercialización se realicen en otros países.

La agroindustria parte de un mejor escenario porque cuenta con una buena dotación de recursos naturales y otros factores básicos (clima, agua). No obstante, enfrenta serios entrampamientos estructurales como: atomización de la tierra, desorganización de los agricultores, desconfianza y falta de cumplimiento de los contratos, baja productividad, producción de calidad heterogénea, débil labor de investigación y transferencia tecnológica, escaso acceso al crédito, por mencionar los más relevantes.
La falta de financiamiento es uno de los principales problemas, pues las instituciones financieras privadas que actualmente otorgan créditos al sector agrario son muy estrictas en cuanto a hectareaje cultivado y propiedad de la tierra, entre otros aspectos (Gobierno Regional de Piura, 2005).

En el caso específico de la producción de limón para exportación, las principales dificultades son las alteraciones climáticas, como el fenómeno de El Niño, cuyos efectos se suelen concentrar en Piura y Lambayeque. Este evento origina una menor producción del fruto, lo que eleva el precio en el mercado interno y puede retrasar las exportaciones. Otro problema radica en las propias características del limón, pues su cáscara gruesa y abundante semilla no favorece su exportación como producto fresco, dadas las preferencias de consumo en otras regiones del mundo. Las variedades de limón más comercializadas en el mercado mundial son los limones sutiles. El limón peruano es idóneo como insumo para la elaboración de jugos y aceites esenciales, por ello una alternativa ventajosa es exportarlo para este uso (Valdivia, 1994: 201).

En la actualidad, los corredores económicos se han convertido en un factor clave para el progreso de países, regiones, ciudades, etcétera, pues crean una dinámica económica competitiva que contribuye a la creación de más mercados regionales, brinda oportunidades de trabajo $\mathrm{y}$, en consecuencia, mejora el ingreso y el bienestar de la población. Con la promoción y el desarrollo de corredores económicos, una región puede tener mayores facilidades para el flujo comercial y migratorio intrarregional. 
Teniendo en cuenta este hecho, la presente investigación estudia la influencia que tienen los corredores económicos para el caso específico del limón de exportación en la región Piura y pretende responder las siguientes interrogantes:

- ¿De qué modo pueden contribuir los corredores económicos al desarrollo de proyectos de articulación productiva-comercial nacional, con proyección al mercado internacional del Asia Pacífico?

- ¿Qué dificultades enfrenta el productor de la sierra en comparación con su par de la costa en la región Piura?

- ¿Cuáles son las posibles alternativas para mejorar la producción de limón con miras a la exportación?

\section{Marco teórico y conceptual}

\subsection{La cooperación económica del Asia Pacífico}

La incorporación del Perú al Foro de Cooperación Económica Asia Pacífico (APEC) en noviembre de 1998 formó parte de una intensa estrategia diplomática iniciada durante los años setenta y supuso la previa incorporación de nuestro país al Consejo Económico de la Cuenca del Pacífico (PBEC) -formado por empresarios privados- en 1990 y al Consejo de Cooperación Económica del Pacífico (PECC) -de carácter gubernamental- en 1991.

APEC es un foro de concertación política orientado a la promoción del comercio, la cooperación, la inversión y el desarrollo económico regional, cuyo fin es establecer una comunidad económica en el Pacífico. No se trata de un bloque regional tradicional, sino de un esquema de integración abierto que busca reducir las barreras comerciales internas para permitir el libre flujo de bienes, servicios, capital y tecnologías, desarrollar métodos de facilitación comercial, financiera y de inversiones y reducir la brecha económica existente entre los países miembros.

La importancia de APEC para el Perú radica no sólo en ser parte del espacio económico más dinámico del mundo en términos de crecimiento -agrupa a países responsables de más de la mitad del comercio y la producción mundial-, sino también en que más del $50 \%$ de nuestro comercio exterior proviene o tiene como destino las economías del Asia Pacífico (Novak, 2003).

En la actualidad, la gran importancia económica de la Cuenca del Pacífico la convierte en un centro de interés mundial. Su localización le permite ser una puerta clave para el vínculo comercial entre países asiáticos y latinoamericanos que no cuentan con costas en el Pacífico pero que buscan ampliar sus mercados. Desde esta perspectiva, el Perú, al ser el único país miembro de la Comunidad Andina (CAN) en formar parte de APEC, se encuentra en una posición estratégica para hacer las veces de interlocutor y promover y desarrollar el intercambio comercial entre ambas regiones (Ministerio de Relaciones Exteriores, 2005).

\subsection{Ventajas comparativas y ventajas competitivas}

La teoría de las ventajas comparativas, formulada por primera vez por David Ricardo a comienzos del siglo XIX, explica los beneficios que se obtiene de la par- 
ticipación en el comercio internacional. Esta aproximación se refiere a la capacidad de una región de producir determinados bienes a un precio relativo menor en comparación con otras regiones. Dada esta situación, la región tenderá a especializarse en la producción y el comercio de una canasta de bienes que mejor pueda producir, bajo parámetros significativos de especialización.

En la economía de hoy, la abundancia de recursos naturales y los salarios bajos ya no son fuentes de ventaja comparativa sostenida. Esto se puede comprobar al observar cómo los patrones de especialización y de desarrollo técnico han permitido a países sin recursos naturales elevar su competitividad y la calidad de vida de sus ciudadanos de modo significativo, tal como ha sucedido y sigue sucediendo en los países del sudeste asiático.

El modelo porteriano de la ventaja competitiva brindó una herramienta para superar el enfoque restrictivo de la ventaja comparativa, que basa la competitividad en la abundancia de recursos naturales y mano de obra barata. En el enfoque de ventaja competitiva, el entorno nacional es decisivo para aumentar la capacidad de las firmas para triunfar en sectores que puedan innovar y producir bienes y servicios de valor agregado para los mercados internos y foráneos.

Mientras las ventajas comparativas se heredan (noción estática), las ventajas competitivas se construyen (noción dinámica); no obstante, las diferencias se agudizan en la medida en que los países y/o sus espacios subnacionales privilegian sólo uno de ambos tipos de ventaja. La evidencia señala que rara vez ha sido po- sible mantener un significativo crecimiento basado únicamente en factores heredados. Este crecimiento ha sido, más bien, producto de la articulación de factores, procesos y elementos de contexto, que configuran condiciones favorables preexistentes y/o inducidas para mejorar la forma de hacer negocios. Algunos de estos factores incluyen empleo de nuevas tecnologías, buenas prácticas empresariales, infraestructura de apoyo a la actividad emprendedora, recurso humano calificado y una combinación valiosa de visiones basadas en la innovación y la transformación de las prácticas de hacer negocio y/o de invertir, con efectos deseables en materia de desarrollo.

Finalmente, en todo lo señalado, al Estado le toca jugar un rol promotor y facilitador a través de factores como la planificación, la inversión en mejora de condiciones dentro de los espacios territoriales con potencial de aprovechamiento productivo, entre otros (Indacochea, 1999: 4-5).

\subsection{Estrategias sistémicas para fortalecer la cooperación en materia productiva}

El empleo práctico de las nociones de ventaja comparativa y ventaja competitiva requiere elaborar e implementar estrategias denominadas sistémicas, ya que estas consideran interrelaciones causaefecto cruzadas entre distintas dimensiones o sistemas de análisis.

Algunas de las estrategias sistémicas que -se sostiene- pueden aportar decisivamente a elevar la competitividad de una actividad microeconómica, como la producción de limón en condiciones exportables, se describen a continuación. 


\section{Cadenas productivas}

Las cadenas productivas son los vínculos que establecen las empresas para diseñar, producir, llevar al mercado, entregar y apoyar a sus productos. La cadena productiva no consiste en realizar las actividades independientemente, sino en buscar relacionarlas de tal manera que se hagan interdependientes. Cada eslabón de la cadena debe ser optimizado y coordinado con los demás para lograr ventajas competitivas (Porter, 1996).

Para lograr adecuados niveles de coordinación entre los agentes participantes de la cadena es necesario establecer acuerdos de competitividad sobre aspectos tecnológicos, empresariales, de recursos humanos, de posicionamiento internacional, etcétera, de manera que el resultado final de toda la cadena sea superior al que cada agente obtendría si actuara aisladamente, es decir, con el único objetivo de maximizar su ganancia sin considerar las sinergias que podrían activarse en el marco de un acuerdo explícito de competitividad.

La cuestión subyacente en este caso es que la competitividad no es sólo el logro particular de una empresa, sino el resultado común de todos los agentes que operan en una región específica, dentro de un determinado sistema productivo.

\section{Cluster o conglomerado}

El cluster o conglomerado es una reunión de empresas que, teniendo en cuenta su cercanía geográfica y el encadenamiento de sus productos, se asocian para maximizar su productividad con altos índices de calidad, a través del trabajo en equipo. Un cluster comprende un conjunto de empresas especializadas a lo largo de una cadena, que puede incluir desde actividades agropecuarias o extractivas hasta manufactureras y de comercialización. El cluster, además, involucra la participación no sólo de las empresas que operan en un espacio determinado, sino también de instituciones de la sociedad civil (gremios, ONG, institutos de investigación, entre otros), que brindan un soporte adecuado para la actividad que se desarrolla, y de instituciones públicas, cuyo rol es proveer un ambiente apropiado para el desarrollo y el crecimiento de los negocios (Porter, 1996).

\section{Corredores económicos}

El concepto de corredor se asocia generalmente a la idea de unir dos puntos distantes entre sí en función de su proyección hacia otros mercados, mediante la mejora del transporte, la energía y las telecomunicaciones, con lo cual se benefician las actividades productivas a lo largo de todo el trayecto del corredor. Se cuida, además, de hacerlo en una forma ambiental y socialmente sostenible (Proyecto PRA, 2001: 6-8).

Los corredores económicos sirven como canales de comercio entre ubicaciones distintas y pueden estar articulados por carreteras, hidrovías o por zonas económicas definidas. Para entender la viabilidad de cada corredor, se le debe analizar en función de su contribución al desarrollo, su grado de preparación y maduración, los proyectos conexos que se puedan ejecutar y su potencial de articulación comercial nacional e internacional (Guerra-García 2004: 6) entre otros.

Históricamente los corredores económicos tuvieron significativo impacto en 
el fortalecimiento de las economías exitosas del Asia Pacífico. Como parte de la estrategia de corredores, la inversión en infraestructura vial generó un impacto positivo en el crecimiento económico y en la reducción de la pobreza, debido a que la inversión permite reducir los costos de producción y los costos de transacción. Es importante considerar que la mayor densidad vial genera incentivos para la especialización, lo que permite una agricultura más intensiva en insumos modernos. Por lo tanto, el impacto de una mejor infraestructura vial en el mayor y mejor acceso a mercados de bienes y en la generación de nuevas oportunidades de empleo debiera posibilitar ganancias de bienestar o riqueza para la región (Gobierno Regional de Piura, 2005).

\section{Los corredores económicos piuranos en este estudio}

\subsection{Corredor económico Sullana- Tambogrande-San Lorenzo-Ayabaca ${ }^{1}$}

Este corredor económico se encuentra integrado por las provincias de Sullana y Ayabaca. Cuenta con un amplio espacio territorial de $11069,26 \mathrm{~km}^{2}$, que representa el 29,7\% del total de la superficie de la región Piura -desde la parte baja de la costa de Sullana hasta la zona alta de la sierra de Ayabaca-. En la parte costera figuran ciudades importantes, como Sullana y Tambogrande, que constituyen ejes clave al servir de nexo para otros distritos de la región, el país y el Ecuador.

Por tradición, la mayor parte de la población del corredor se ha dedicado a la

1. Acápite basado en Albirena Eyzaguirre, 2004. actividad agropecuaria: arroz, algodón, frutales y ganadería de pequeña y mediana escala. Sin embargo, estos productos contienen escaso valor agregado, situación que se acentúa en la sierra, que carece de infraestructura de riego. En cambio Sullana sí cuenta con recursos hídricos por el Proyecto Chira-Piura; y las zonas de Tambogrande y Las Lomas, con el reservorio de San Lorenzo. La actividad comercial (de productos e insumos) es la segunda en importancia en términos de absorción de mano de obra y gira en torno de la agricultura. Otra actividad es la manufactura, con mayor dinamismo en la provincia de Sullana y Tambogrande, que se explica por la operación de empresas agroindustriales de frutas, bebidas y de aceite de limón, constituidas gracias a la presencia de infraestructura y servicios básicos.

Entre los flujos comerciales que se generan en el corredor pueden mencionarse los siguientes: la producción del valle del Chira se destina al mercado regional y a Ecuador; San Lorenzo produce mango con fines de exportación, el que sale por el puerto de Paita; el banano orgánico de Querecotillo se comercializa en Lima y Ecuador; el limón producido en Cieneguillo es llevado a Piura y Lima. Asimismo, lo producido desde la zona de Suyo hasta Ayabaca -arroz, café, caña de azúcar y ganado- se comercializa en Piura, Lima y Ecuador. Los productos agroindustriales -bebidas gaseosas, jugos y néctares- se comercializan en el mercado nacional, y el aceite esencial de limón se destina al mercado internacional.

Del análisis de los factores clave para la competitividad, se desprende que el medio natural es favorable para el desarrollo de actividades agrícolas y pecuarias, 
por la existencia de tierras de buena calidad, microclimas y sistema de agua regulada. Sin embargo, uno de los elementos negativos es la sedimentación de los reservorios, que afecta no sólo al corredor, sino a gran parte de la superficie agrícola de la región. En términos de infraestructura, resalta el Proyecto Chira-Piura, por su trascendencia para la región. Asimismo, este es el corredor que cuenta con mayor kilometraje de red vial asfaltada, debido al eje costero de la carretera Panamericana Norte, la cual hace posible el rápido acceso a los puertos de Paita y Talara y a la zona de frontera. Por el contrario, los centros poblados del interior cuentan sólo con trochas y caminos de herradura, situación muy acentuada en la zona de sierra.

Respecto a los servicios no financieros, son notorias la escasa asistencia técnica y la poca capacitación, brindadas sólo por las empresas exportadoras. Casi no existen centros de investigación y desarrollo tecnológico, limitación que se espera superar con el recientemente creado Centro de Formación Profesional Binacional, que ofrece las carreras de producción y negocios agropecuarios.

En el ámbito de la salud, la ciudad de Sullana concentra la mayoría de establecimientos de atención y la mayor cobertura del servicio, mientras que en la sierra de Ayabaca la red de establecimientos es insuficiente; y el servicio prestado, muy limitado. Lo mismo sucede con los servicios básicos -agua, alcantarillado, energía-, cuya carencia es notoria en los centros poblados de la sierra.

Asimismo, son escasos los mecanismos de información para los productores, quienes no tienen conocimiento del mer- cado ni de oportunidades de negocios. En lo que se refiere a los servicios financieros, también hay desequilibrio en el corredor, ya que la mayor cantidad de establecimientos del sistema bancario y no bancario, y por lo tanto la mayor oferta de recursos, se concentra en la provincia de Sullana.

Finalmente, los patrones culturales se muestran débiles; destacan la falta de identidad y la desconfianza de la población, a causa del fracaso de experiencias de organización y asociativismo.

\subsection{Corredor económico Chulucanas- Morropón-Huancabamba ${ }^{2}$}

Otro de los corredores económicos identificados en la región comprende desde la parte baja de Chulucanas y Morropón hasta la parte alta de la provincia de Huancabamba. En conjunto, este corredor representa el $26,8 \%$ del total de la superficie regional, y en el 2002 contaba con una población de 367877 habitantes. La provincia de Morropón, ubicada en el valle del Alto Piura, se caracteriza por sus tierras fértiles, clima favorable y bosques secos, factores positivos para el desarrollo de la actividad agropecuaria. La ciudad de Chulucanas constituye el eje articulador del corredor, gracias a su ubicación geográfica y a que cuenta con vías de acceso directas a la capital del departamento y al resto del país. Es el centro de acopio y comercialización de la producción agrícola, así como el centro de la producción y comercio de artesanía.

En cuanto a la actividad agrícola, en el corredor se producen mango, plátano y limón, que se comercializan en Piura,

2. Acápite basado en Albirena Eyzaguirre, 2004. 
Chiclayo y Lima; café de Huancabamba, que es comercializado en el mercado exterior y también llevado a la provincia de San Ignacio por la zona de El Carmen de la Frontera; maíz, arroz, frejol y papa, que se venden a mayoristas y transportistas y son distribuidos en los mercados regional y nacional. En la provincia de Huancabamba se produce, en menor escala, trigo, granadilla y cacao, destinados a los mercados local y regional. En lo que se refiere a la actividad pecuaria, el ganado producido, principalmente en el distrito de La Matanza, es llevado a Chiclayo y, en menor medida, a Piura. Otra actividad desarrollada en este corredor es la agroindustria, en la que destacan la elaboración de miel de abeja, algarrobina y queso (producido en Sapalache y comercializado en los mercados local y regional), así como la producción de cochinilla, panela granulada y plantas medicinales.

Respecto de la artesanía, ha ganado renombre la cerámica de Chulucanas, de muy reconocida calidad en los ámbitos nacional e internacional; sobresalen también la alfarería, la elaboración de textiles -prendas de vestir, tejidos de lana, confección de telas y ponchos- y también de sogas y hamacas. La actividad turística tiene como principal atractivo las lagunas de Las Huaringas, ubicadas en la parte alta de la provincia de Huancabamba, a cuyas aguas se les atribuye propiedades medicinales.

Si se analizan los factores básicos para la competitividad, se puede decir que el medio natural del corredor es favorable para la agricultura, por la variedad de microclimas, que permite una diversidad de cultivos en los diferentes pisos altitudinales, y por la abundante vegetación: cultivos, pastos y bosques. Un aspecto limi- tante es la escasez de agua, que causa problemas de riego.

La infraestructura constituye un factor en contra, por el mal estado de la red vial, que dificulta el acceso a centros poblados, principalmente en la zona de sierra, sobre todo en época de lluvia. Asimismo, hay dificultad para la captación de agua de los afluentes para el riego de los cultivos debido a la falta de infraestructura. El último fenómeno de El Niño agravó la situación, pues propició el desvío del cauce del río y colapsaron las tomas de captación. En la zona de la sierra es muy baja la capacidad de generación eléctrica, por ello la mayoría de viviendas del área rural carecen de electricidad. Asimismo, el acceso a medios de comunicación es muy limitado, situación que se agudiza en pueblos alejados de la sierra, donde el medio de comunicación más utilizado es la radio.

En cuanto a los servicios de apoyo a la producción, es escasa la asistencia técnica y la capacitación para los productores, excepto los cafetaleros. El acceso a información de mercados sigue siendo un problema relevante. Respecto a los servicios financieros, hay instituciones que brindan crédito a los productores, pero acceder a este es difícil. La mayoría opta por el crédito comercial y el informal.

\section{Objetivos, hipótesis y planteamiento del modelo}

El objetivo general de esta investigación es estudiar si los corredores económicos han contribuido a la mejora de la producción de limón y al desarrollo de la región durante el periodo comprendido entre enero del 2002 y setiembre del 2004. 
Los objetivos específicos son dos:

- Describir las ventajas más importantes de los corredores económicos e identificar los problemas y las limitaciones asociados con su desarrollo y con el desarrollo del sector agrario nacional entre los años 2002 y 2004.

- Analizar si se han tomado en cuenta las inversiones en infraestructura vial, la capacitación de los agricultores y otras medidas necesarias para el desarrollo de los corredores económicos y definir las alternativas de solución a la problemática existente, así como su viabilidad y el impacto sobre la región Piura entre los años 2002 y 2004.

\section{Hipótesis general}

La falta de inversiones adecuadas para el desarrollo de los corredores económicos impide el crecimiento de la producción de limón. Con la mejora y el uso de los corredores habrá más incentivos para realizar diferentes tipos de actividades productivas que contribuirán a la mejora de la región durante el periodo de estudio.

\section{Hipótesis específicas}

La implementación de los corredores generará un impacto positivo en el crecimiento económico de las regiones siempre y cuando se apliquen mecanismos, políticas y estrategias adecuadas para aumentar la inversión y fomentar la integración regional, lo que contribuirá a incrementar los ingresos de los productores, con la consecuente generación de divisas.

En el periodo en estudio, la producción de limón en Piura se ve afectada significativamente por el precio promedio y por el precio en chacra del fruto. Sin embargo, no ha sido muy afectada por los corredores económicos, por los estándares de producción del pasado ni por el PBI agrícola, lo cual se debe a la poca inversión en infraestructura, la conservación de una estructura agraria atrasada y el mantenimiento de políticas agrarias que no permiten el desarrollo del sector.

Se utiliza como principales variables el volumen de producción de limón en la región Piura (INEI, 2005a) durante el periodo en estudio, así como la inversión en infraestructura vial (MTC, 2005), el precio promedio mensual del limón (INEI, 2005a) que compone la canasta del índice de precios al por mayor 2003-2004, el precio promedio en chacra (INEI, 2005a) y el producto bruto interno agrícola (INEI, 2005b).

En cuanto a la primera hipótesis específica, se elabora una matriz de evaluación del factor externo (EFE), herramienta que resume y evalúa la información económica, social, cultural, demográfica, ambiental, política, gubernamental, legal, tecnológica y competitiva.

Con respecto a la segunda hipótesis específica, se diseña un modelo econométrico en series de tiempo (dinámica), en el cual la variable objetivo es explicada a través de la acción de otras variables. También se detallan las variables predeterminadas, que son causas o componentes y las responsables de la variación de la variable endógena. Mediante esta metodología, se estudiarán las pruebas de relevancia global, que evalúan el impacto e indican la explicatividad de todos los regresores en conjunto sobre la variable endógena y luego se analizarán las pruebas de relevancia individual, que son in- 
dicadoras de la respuesta de la variable endógena ante variaciones individuales de cada uno de los regresores (variables predeterminadas).

\section{Comprobación de la primera hipótesis}

Esta hipótesis se comprobará a través de la matriz de evaluación de factor externo (EFE). Es importante señalar que conforme haya una mejora en la infraestructura vial, se contribuirá al progreso de la región.
Matriz de evaluación de factor externo (EFE)

La matriz (EFE) nos permite identificar las oportunidades y amenazas que afectan a la región. En este caso, las oportunidades se relacionan con el desarrollo de corredores económicos y la mejora de la infraestructura vial.

Un valor ponderado total de 4,0 indica que la empresa responde de manera sorprendente a las oportunidades y amenazas presentes en su sector; en otras palabras,

Factores externos clave

Valor Clasificación

Valor

ponderado

\section{OPORTUNIDADES}

1. Acceso a mercados internacionales como el del Asia Pacífico.

0,15

4

0,60

2. Crecimiento económico y social.

Descentralización.

$\begin{array}{lll}0,15 & 4 & 0,60 \\ 0,10 & 4 & 0,40 \\ 0,10 & 3 & 0,30 \\ 0,10 & 3 & 0,30\end{array}$

3. Desarrollo de servicios básicos.

4. Generación de empleo.

5. Facilidades de flujo comercial y migratorio.

0,10

0,30

AMENAZAS

1. Cambios en los factores climáticos.

$0,05 \quad 2 \quad 0,10$

2. Inestabilidad política.

$0,05 \quad 2 \quad 0,10$

3. Incipiente investigación y desarrollo.

0,05

0,05

4. Carencia de infraestructura vial adecuada.

0,15

1

0,15

5. Falta de coordinación en el accionar de entidades del sector agrario y sus organismos desconcentrados públicos y privados.

0,10

1

0,10

Total

1,00

2,70

Elaboración propia. 
las estrategias de la empresa aprovechan en forma eficaz las oportunidades existentes y reducen al mínimo los efectos adversos potenciales de las amenazas externas. Un puntaje total de 1,0 significa que las estrategias de la empresa no aprovechan las oportunidades ni evitan las amenazas externas (Fred, 2003).

En este caso, el resultado de 2,70 quiere decir que la región intenta aprovechar las oportunidades generadas por el desarrollo de la infraestructura vial y reducir al mínimo los efectos potenciales de las amenazas. Por lo tanto, la construcción y el mantenimiento de las redes viales posibilitarán la mejora del acceso a los servicios básicos, el logro de una mayor integración y la articulación de las poblaciones, así como la generación de más puestos de trabajo y el acceso a más mercados para el desarrollo de la propia agricultura. De esta manera, los productores tendrán la oportunidad de ofrecer sus productos a mercados como el del Asia Pacífico, que concentra más de un tercio de la población mundial. Pero para ingresar a este inmenso mercado debe cumplirse todas las normas establecidas, mantener un Estado democrático y una administración de justicia autónoma y respetar los derechos humanos y la libertad de expresión.

\section{Comprobación de la segunda hipótesis}

Planteamiento del modelo

Relación base

LIMONPI $=f$ (INFRAESTRUCTURA, PBIAGRICOLA, PPRO, PCHACRA)

\section{Especificación del modelo}

$$
\begin{aligned}
& \Delta \text { LIMONPI }_{\mathrm{t}}=\alpha+\beta_{1} \Delta \text { INFRAESTRUCTURA }_{\mathrm{t}} \\
& +\beta_{2} \Delta \text { PBIAGRICOLA }_{\mathrm{t}}+\beta_{3} \Delta \text { PPRO }_{\mathrm{t}}+ \\
& \beta_{4} \Delta \text { PCHACRA }_{\mathrm{t}}+\beta_{5} \Delta \text { LIMONPI }_{\mathrm{t}-1}+ \\
& \beta_{6} \Delta \text { INFRAESTRUCTURA }_{\mathrm{t}-1}+ \\
& \beta_{7} \Delta \text { PBIAGRICOLA }_{\mathrm{t}-1}+\mathrm{E}_{\mathrm{t}}
\end{aligned}
$$

\begin{tabular}{|c|c|c|c|c|}
\hline Notación & Tipo de variable & Significado & Unidad & Fuente \\
\hline LIMONPI & Endógena & $\begin{array}{l}\text { Producción } \\
\text { de limón en Piura. }\end{array}$ & Toneladas & INEI \\
\hline INFRAESTRUCTURA & Exógena & $\begin{array}{l}\text { Inversión en } \\
\text { infraestructura vial. }\end{array}$ & Nuevos soles & $\begin{array}{l}\text { MTC Provías } \\
\text { Nacional }\end{array}$ \\
\hline PPRO & Exógena & $\begin{array}{l}\text { Precio promedio mensual } \\
\text { que compone la canasta } \\
\text { del índice de precios } \\
\text { al por mayor } 2003-2004\end{array}$ & $\begin{array}{l}\text { Nuevos soles } \\
\text { por kilogramo }\end{array}$ & INEI \\
\hline PCHACRA & Exógena & $\begin{array}{l}\text { Precio promedio } \\
\text { en chacra }\end{array}$ & $\begin{array}{l}\text { Nuevos soles } \\
\text { por kilogramo }\end{array}$ & INEI \\
\hline PBIAGRICOLA & Exógena & $\begin{array}{l}\text { Producto bruto interno } \\
\text { agrícola }\end{array}$ & $\begin{array}{l}\text { Millones de } \\
\text { nuevos soles a } \\
\text { precios de } 1994\end{array}$ & INEI \\
\hline
\end{tabular}

Descripción de las variables 


\section{Resultado del modelo}

Dependent Variable: D(LIMONPI)

Method: Least Squares

Date: 10/14/05 Time: 19:19

Sample (adjusted): 2002M03 2004M09

Included observations: 31 after adjustments

\begin{tabular}{lrccc}
\hline Variable & Coefficient & Std. Error & t-Statistic & Prob. \\
\hline C & $-0,330018$ & 0,443875 & $-0,743494$ & 0,4647 \\
D(INFRAESTRUCTURA) & $-5,73 E-07$ & $4,86 E-07$ & $-1,180443$ & 0,2499 \\
D(PBIAGRICOLA) & $-0,001588$ & 0,003614 & $-0,439444$ & 0,6644 \\
D(PPRO) & $-18,13018$ & 5,565089 & $-3,257842$ & 0,0035 \\
D(PCHACRA) & $-6,251261$ & 1,668778 & $-3,746010$ & 0,0011 \\
D(LIMONPI(-1)) & $-0,248440$ & 0,195293 & $-1,272145$ & 0,2160 \\
D(INFRAESTRUCTURA(-1)) & $3,16 E-08$ & $5,88 E-07$ & 0,053692 & 0,9576 \\
D(PBIAGRICOLA(-1)) & 0,000229 & 0,003605 & 0,063596 & 0,9498 \\
\hline R-squared & 0,501773 & Mean dependent var & $-0,260968$ \\
Adjusted R-squared & 0,350139 & S,D, dependent var & 2,985652 \\
S.E. of regression & 2,406852 & Akaike info criterion & 4,812152 \\
Sum squared resid & 133,2375 & Schwarz criterion & 5,182214 & \\
Log likelihood & $-66,58836$ & F-statistic & 3,309103 & \\
Durbin-Watson stat & 2,021139 & Prob (F-statistic) & 0,013838 & \\
\hline
\end{tabular}

\section{Análisis de resultados}

Dentro de las pruebas de relevancia individual, el coeficiente de determinación $\mathrm{R}^{2}$ señala que hay un $50 \%$ de explicatividad de las variables predeterminadas con relación a la variable endógena y que otro
$50 \%$ (el complemento) es explicado por lo estocástico. También se tiene la prueba de Fisher $(\mathrm{F})$, cuyo resultado $=0,013$ indica que hay significancia global de todas las variables predeterminadas con respecto a la variable endógena.

\section{Prueba de normalidad de los errores de Jarque y Bera (JB)}

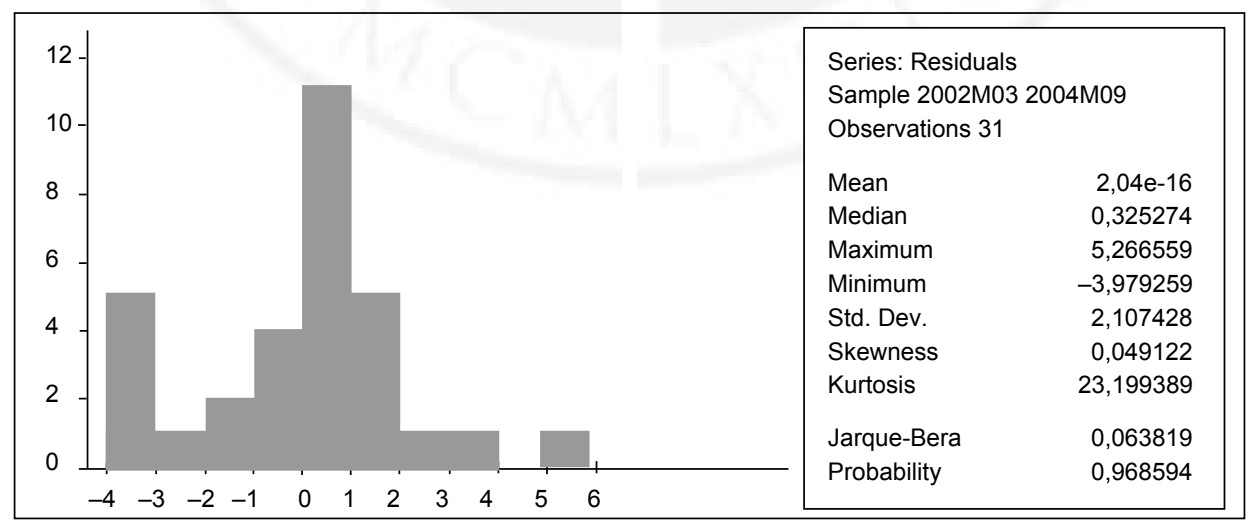


Lo ideal es que la Kurtosis se aproxime a 3, pero en este caso la Kurtosis $\approx 3,19$. Sin embargo, no es muy elevada y, por lo tanto, no hay aplicación de volatilidad.

Probabilidad $=0,968594>0,05 \rightarrow$ Se acepta la hipótesis nula $\left(\mathrm{A} \mathrm{H}_{\mathrm{O}}\right)$.

Asimetría $=$ Skewness $\approx 0,04$, tiene asimetría.

Para lograr una distribución normal perfecta, el valor del estadístico Jarque-Bera (JB) tendría que ser $=0$. Por lo tanto, lo que se busca es que el valor del estadístico JB sea lo más bajo posible, ya que se analiza variables económicas. En este caso el estadístico $\mathrm{JB}=0,06 \approx 0$.

$\mathrm{H}_{\mathrm{O}}$ : Los errores ex post pueden aproximarse a la distribución normal. Por lo tanto, hay anormalidad pero no sustantiva.

$\mathrm{H}_{1}$ : Los errores ex post no pueden aproximarse a la distribución normal. Por lo tanto, hay anormalidad pero muy fuerte.

Decisión $\mathrm{A} \mathrm{H}_{\mathrm{O}}$ : Como no hay tanta anormalidad se puede estimar por mínimos cuadrados.

\section{Análisis de diferencias}

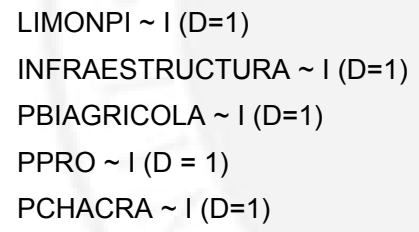

El modelo se encuentra cointegrado. Es una ecuación de cointegración a niveles, dado que el residual de dicha ecuación es estacionario a niveles y porque dicha ecuación no tiene autocorrelación en defecto. La cointegración de dos o más series de tiempo sugiere que existe una relación de largo plazo o de equilibrio entre ellas.

Null Hypothesis: E has a unit root

Exogenous: None

Lag Length: 0 (Automatic based on AIC, MAXLAG=0)

\begin{tabular}{lccc}
\hline & & t-Statistic & Prob. $^{*}$ \\
\hline Augmented Dickey-Fuller test statistic & $-5,019833$ & \multirow{2}{*}{0,0000} \\
Test critical values: & $1 \%$ level & $-2,644302$ & \\
& $5 \%$ level & $-1,952473$ & \\
& $10 \%$ level & $-1,610211$ & \\
\hline
\end{tabular}

*MacKinnon (1996) one-sided p-values. 
Augmented Dickey-Fuller Test Equation

Dependent Variable: $\mathrm{D}(\mathrm{E})$

Method: Least Squares

Date: 10/16/05 Time: 23:14

Sample (adjusted): 2002M04 2004M09

Included observations: 30 after adjustments

\begin{tabular}{lclrr}
\hline Variable & Coefficient & Std. Error & t-Statistic & Prob. \\
\hline E(-1) & $-0,927496$ & 0,184766 & $-5,019833$ & 0,0000 \\
\hline R-squared & 0,464905 & Mean dependent var & $-0,021030$ \\
Adjusted R-squared & 0,464905 & S,D, dependent var & 2,985368 \\
S.E. of regression & 2,183803 & Akaike info criterion & 4,432778 \\
Sum squared resid & 138,3009 & Schwarz criterion & 4,479485 \\
Log likelihood & $-65,49167$ & Durbin-Watson stat & 1,799876
\end{tabular}

$e \sim l(D=0)$

\section{Análisis de la segunda hipótesis}

Podemos ver en los test de BreuschGodfrey que no hay autocorrelación significativa. Además, la t-Student nos indica que la inversión en infraestructura en el periodo actual y en los periodos anteriores afecta de manera poco significativa la producción de limón en Piura. Esto se debe a la centralización, la falta de inversiones gubernamentales en algunos sectores de la región y a la falta de inversión en infraestructura básica, ya que no existe una adecuada coordinación en el manejo de políticas para la mejora de la región.

Con respecto a la causalidad, cuando existe una menor inversión en infraestructura, la producción de limón disminuye; y cuando hay una mayor inversión en in- fraestructura, aumenta la producción de limón. Esto debido a las mejoras en carreteras, lo que permite llegar a mercados anteriormente inaccesibles. Sin embargo, este efecto se observa cuando la inversión en infraestructura se realiza en periodos anteriores, pues la inversión en infraestructura actual no va a influir en la producción existente, sino en las futuras, cuando ya esté operativa una carretera, un puente, entre otras obras civiles. Por ese motivo, la inversión en periodos anteriores tiene un coeficiente positivo, porque está influyendo en la producción actual; en cambio, la inversión en infraestructura del periodo vigente tiene un coeficiente negativo, porque puede perjudicar la producción actual debido a que las obras de construcción tal vez se conviertan en un obstáculo para el desarrollo de las actividades agropecuarias.

\section{Test Breusch-Godfrey}

Breusch-Godfrey Serial Correlation LM Test:

$\begin{array}{llll}\text { F-statistic } & 0,007843 & \text { Probability } & 0,930234 \\ \text { Obs*R-squared } & 0,011047 & \text { Probability } & 0,916293\end{array}$


T-STUDENT

\begin{tabular}{ccccc}
\hline Variable & Coefficient & Std. Error & t-Statistic & Prob. \\
\hline D(INFRAESTRUCTURA) & $-5,73 \mathrm{E}-07$ & $4,86 \mathrm{E}-07$ & $-1,180443$ & 0,2499 \\
\hline
\end{tabular}

$\mathrm{H}_{\mathrm{O}}: \beta_{1}=0$, la incidencia de D(INFRAESTRUCTURA) en la endógena no es significativa. $\mathrm{H}_{1}: \beta_{1} \neq 0$, la incidencia de D(INFRAESTRUCTURA) en la variable endógena sí es significativa.

\section{T-STUDENT}

\begin{tabular}{ccccc}
\hline Variable & Coefficient & Std. Error & t-Statistic & Prob. \\
\hline D(INFRAESTRUCTURA(-1)) & $3,16 \mathrm{E}-08$ & $5,88 \mathrm{E}-07$ & 0,053692 & 0,9576 \\
\hline
\end{tabular}

$\mathrm{H}_{\mathrm{O}}: \beta_{6}=0$, la incidencia de D(INFRAESTRUCTURA $\left.)(-1)\right)$ en la variable endógena no es significativa.

$\mathrm{H}_{1}: \beta_{6} \neq 0$, la incidencia de D(INFRAESTRUCTURA(-1)) en la variable endógena sí es significativa.

De igual modo, la t-Student indica que el precio promedio mensual que compone la canasta del índice de precios al por mayor 2003-2004 y el precio en chacra afectan de manera significativa la producción de limón en Piura. Esto se debe al interés de los agricultores en adaptarse al nuevo entorno de la economía, al afán de adquirir conocimientos sobre el mercado y las oportunidades que este genera.

En este caso, la causalidad se produce cuando hay un aumento de la demanda de limón, pues se incrementan los precios y esto motiva a los productores a incrementar la producción para satisfacer las necesidades de los consumidores. Sin embargo, la alteración de las condiciones climáticas (fenómeno de El Niño, lluvias torrenciales y sequías), sobre todo en los meses de verano, origina una menor producción de la fruta, lo que se traduce en un incremento de los precios en el mercado interno y retrasa la campaña comercial de las exportaciones. Motivo por el cual hay una relación inversa entre la producción de limón y los precios promedio y los precios de chacra.

T-STUDENT

\begin{tabular}{ccccc}
\hline Variable & Coefficient & Std. Error & t-Statistic & Prob. \\
\hline $\mathrm{D}(\mathrm{PPRO})$ & $-18,13018$ & 5,565089 & $-3,257842$ & 0,0035 \\
\hline
\end{tabular}

$\mathrm{H}_{\mathrm{O}}: \beta_{3}=0$, la incidencia de $\mathrm{D}(\mathrm{PPRO})$ en la variable endógena no es significativa. $\mathrm{H}_{1}: \beta_{3} \neq 0$, la incidencia de $\mathrm{D}(\mathrm{PPRO})$ en la variable endógena sí es significativa. 
T - STUDENT

\begin{tabular}{lllll}
\hline VariableCoefficient & Std. Error & t-Statistic & Prob. & \\
\hline D(PCHACRA $)$ & $-6,251261$ & 1,668778 & $-3,746010$ & 0,0011 \\
\hline
\end{tabular}

$\mathrm{H}_{\mathrm{O}}: \beta_{4}=0$, La incidencia de $\mathrm{D}$ (PCHACRA) en la variable endógena no es significativa. $\mathrm{H}_{1}: \beta_{4} \neq 0$, La incidencia de $\mathrm{D}$ (PCHACRA) en la variable endógena sí es significativa.

Por otra parte, la t-Student indica que los estándares de producción del limón anteriores al periodo de estudios no afectan de manera significativa la producción de limón en Piura y tampoco lo hacen ni el PBI agrícola actual ni el PBI agrícola anterior. Esto se debe a que el país mantiene una estructura agraria atrasada y políticas agrarias inadecuadas que no permiten el desarrollo del sector. Además, el apoyo al agro no ha sido integral, motivo por el cual no se ha fomentado la producción ni la productividad. A ello también se agregan los efectos de los desastres originados por el fenómeno de El Niño.

Con relación a la causalidad, cuando crece el PBI agrícola hay una mejora en todos sus componentes. Por lo tanto, habría una mayor producción de limón, parte integrante del PBI agrícola. Si la producción de limón anterior ha sido buena, entonces afectará la producción actual, porque los agricultores serán más optimistas y tratarán de incrementar el cultivo constantemente.

T-STUDENT

\begin{tabular}{ccccc}
\hline Variable & Coefficient & Std. Error & t-Statistic & Prob. \\
\hline $\mathrm{D}($ LIMONPI(-1)) & $-0,248440$ & 0,195293 & $-1,272145$ & 0,2160 \\
\hline
\end{tabular}

$\mathrm{H}_{\mathrm{O}}: \beta_{5}=0$, la incidencia de $\mathrm{D}($ LIMONPI(-1)) en la variable endógena no es significativa. $\mathrm{H}_{1}: \beta_{5} \neq 0$, la incidencia de D(LIMONPI(-1)) en la variable endógena sí es significativa.

T-STUDENT

\begin{tabular}{ccccc}
\hline Variable & Coefficient & Std. Error & t-Statistic & Prob. \\
\hline D(PBIAGRICOLA) & $-0,001588$ & 0,003614 & $-0,439444$ & 0,6644 \\
\hline
\end{tabular}

$\mathrm{H}_{\mathrm{O}}: \beta_{2}=0$, la incidencia de $\mathrm{D}$ (PBAIGRICOLA) en la variable endógena no es significativa. $\mathrm{H}_{1}: \beta_{2} \neq 0$, la incidencia de D(PBAIGRICOLA) en la variable endógena sí es significativa.

\section{T-STUDENT}

\begin{tabular}{ccccc}
\hline Variable & Coefficient & Std. Error & t-Statistic & Prob. \\
\hline $\mathrm{D}(\mathrm{PBIAGRICOLA}(-1))$ & 0,000229 & 0,003605 & 0,063596 & 0,9498 \\
\hline
\end{tabular}

$\mathrm{H}_{\mathrm{O}}: \beta_{7}=0$, la incidencia de $\left.\mathrm{D}(\mathrm{PBAIGRICOLA})(-1)\right)$ en la endógena no es significativa. $\mathrm{H}_{1}: \beta_{7} \neq 0$, la incidencia de $\left.\mathrm{D}(\mathrm{PBAIGRICOLA})(-1)\right)$ en la endógena sí es significativa. 


\section{Conclusiones}

La Cuenca del Pacífico tiene una gran importancia económica que la convierte en un centro de interés mundial. Su localización permite que esta región sea una importante puerta de vinculación comercial entre países asiáticos y latinoamericanos que no cuentan con costas en el Pacífico pero que buscan ampliar sus mercados. Desde esta perspectiva, el Perú, al ser el único país miembro de la Comunidad Andina (CAN) en formar parte de APEC, se encuentra ubicado en una posición estratégica que le permite hacer las veces de interlocutor para promover y desarrollar el intercambio comercial entre ambas regiones.

Los corredores están vinculados a los países con economías fuertes, como la región Asia Pacífico, y las economías están relacionadas con los países vecinos. La inversión en infraestructura vial genera un impacto positivo en el crecimiento económico y en la reducción de la pobreza. Los corredores propician mercados para el desarrollo de la agricultura y crean oportunidades en términos de mercados de bienes, de trabajo, de capital, etcétera.

La problemática que acompaña al tema de la producción de limón abarca principalmente a los pequeños y medianos agricultores. La falta de grandes inversiones gubernamentales, los altos costos de transacción, la falta de infraestructura básica, la baja calidad de los recursos humanos y naturales y, por último, la distancia hacia los mercados hacen que la producción no sea totalmente óptima.

\section{Recomendaciones}

La región Piura debe aprovechar que el Perú es el único país de la Comunidad Andina miembro de APEC. En este sentido, debe convertirse en enlace entre las economías andinas y asiáticas fomentando el intercambio comercial, la inversión y la cooperación entre ambos bloques. En este punto es necesario insistir en que nuestras exportaciones al Asia no pueden seguir limitándose al ámbito de las materias primas, sino que deben incorporar mayor valor agregado.

El mantenimiento de las redes viales hará posible la mejora del acceso a los servicios básicos, el logro de una mayor integración y la articulación de las poblaciones, así como la generación de más puestos de trabajo.

Un mecanismo para romper con la debilidad del eslabón agrícola es el establecimiento de alianzas entre agroindustrias y productores, por medio de las cuales las primeras proporcionen a los segundos asistencia financiera y técnica y mercados asegurados, mientras que los segundos se comprometan a cumplir las recomendaciones técnicas correspondientes y a entregar la cosecha al final de la campaña.

Por último, es importante fomentar la integración y articulación de las comunidades y de la población rural a la dinámica del desarrollo regional y nacional, a través de la implementación y fortalecimiento de una formación profesional de calidad que identifique y dé respuesta a las actuales y futuras demandas con participación activa del Estado, el sector privado, los trabajadores y la sociedad civil. 


\section{Bibliografía y fuentes}

ALBIRENA EYZAGUIRRE, Luis. 2004a. La competitividad del corredor económico: Sullana-Tambogrande-San Lorenzo-Ayabaca. El Tiempo. Piura, 18 de mar.

—_. 2004b. La competitividad del corredor económico: Chulucanas-Morropón y Huancabamba. El Tiempo. Piura, 25 de mar.

DIARIO GESTIÓN. 2005. Especial de la región Piura. Lima, 20 de abr.

FRED, David. 2003. Conceptos de administración estratégica. $9^{\mathrm{a}}$ ed. México: Pearson Education.

GUERRA-GARCÍA, Gustavo. 2004. Infraestructura para los corredores. Semana Económica. Lima, nov., n. ${ }^{\circ} 945$.

INDACOCHEA, Alejandro. 2000. La promoción-país y el desarrollo de ventajas competitivas: un nuevo rol del Estado para la descentralización del país. En: Boza, Beatriz (ed.). El rol del Estado en la labor de promoción-país: hacia una auditoría académica de PromPerú. Lima: PromPerú. Págs. 241-264.

- 1999. Competitividad regional. Gerencia. Lima, mar., n. ${ }^{\circ}$ 237, págs. 5-6.

NOVAK , Fabián. 2003. Proyección del Perú en APEC. Quehacer. Lima, n. ${ }^{\circ} 131$.

PERÚ. GOBIERNO REGIONAL DE PIURA. 2005. Programa regional de competitividad de Piura 2004-2010. Mayo [www.interregionesperu.org].
PERÚ. INEI. 2002. Avance económico y social regional: Piura [www.inei.gob.pe].

PERÚ. INEI. 2005a. Avance económico y social regional de Piura, setiembre 2004. Lima, oct.

2005b. Información económica, sector real de la producción. Lima, oct.

PERÚ. MINISTERIO DE AGRICULTURA. 2005. Glosario.

PERÚ. MINISTERIO DE COMERCIO EXTERIOR. 2005. Preguntas y respuestas sobre el TLC Perú-EE.UU. Lima, mar. [www.mincetur.gob.pe].

PERÚ. MINISTERIO DE LA PRODUCCIÓN. 2005. Plan de competitividad productiva. Lima, mar. [www.produce.gob.pe].

PERÚ. MINISTERIO DE RELACIONES EXTERIORES. 2005. Importancia de la participación del Perú en el APEC. Lima, set. [www.rree.gob.pe].

PERÚ. MINISTERIO DE TRANSPORTES Y COMUNICACIONES. PROVÍAS NACIONAL. 2005. Infraestructura vial de Piura 2002-2004. Lima, MTC, set.

PERX PIURA. 2004. Plan estratégico exportador de Piura. Jun. [www.mincetur.gob.pe].

PIURA ON LINE. Centro de Documentación Regional CEDIR-Cipca. [www.region piura.gob.pe]. 
PORTER, Michael. 1990. Las ventajas competitivas de las naciones. Buenos Aires: Vergara.

—_. 1996. Ventaja competitiva: creación y sostenimiento de un desempeño superior. México: Continental.
PROYECTO DE REDUCCIÓN Y ALIVIO A LA POBREZA (PRA). 2001. La estrategia de corredores económicos y el proyecto de reducción y alivio a la pobreza. Boletín informativo. Lima, set.

VALDIVIA, Martín. 1994. Comercialización agrícola en el Perú. Lima, Grade. 


\section{Anexo 1. Relación de causalidad: árbol de problemas}

\begin{tabular}{|c|c|c|c|}
\hline $\begin{array}{l}\text { Efecto } \\
\text { principal }\end{array}$ & \multicolumn{3}{|c|}{$\begin{array}{l}\text { La insuficiente inversión en la región Piura } \\
\text { afecta la producción del sector. }\end{array}$} \\
\hline $\begin{array}{l}\text { Efectos } \\
\text { indirectos }\end{array}$ & $\begin{array}{l}\text { La poca promoción de la } \\
\text { inversión en } \\
\text { infraestructura física } \\
\text { imposibilita la prestación } \\
\text { de servicios para el } \\
\text { comercio internacional. }\end{array}$ & $\begin{array}{l}\text { Restricciones en el } \\
\text { uso de los servicios } \\
\text { de salud y de } \\
\text { educación. }\end{array}$ & $\begin{array}{l}\text { Los productores son } \\
\text { diestros en el manejo de } \\
\text { cultivos tradicionales, pero } \\
\text { carecen de conocimientos } \\
\text { sobre el mercado y sus } \\
\text { oportunidades. }\end{array}$ \\
\hline $\begin{array}{l}\text { Efectos } \\
\text { directos }\end{array}$ & $\begin{array}{l}\text { No se ha construido un } \\
\text { entorno competitivo, } \\
\text { debido a la ineficiencia } \\
\text { administrativa y a la débil } \\
\text { gobernabilidad } \\
\text { democrática. }\end{array}$ & $\begin{array}{l}\text { Crédito escaso y de } \\
\text { difícil acceso } \\
\text { (especialmente para } \\
\text { la pequeña } \\
\text { agricultura). }\end{array}$ & $\begin{array}{l}\text { Débil potenciación de } \\
\text { los corredores } \\
\text { económico-sociales. }\end{array}$ \\
\hline $\begin{array}{l}\text { Problema } \\
\text { central }\end{array}$ & \multicolumn{3}{|c|}{$\begin{array}{c}\text { El escaso desarrollo regional y competitivo compromete } \\
\text { el bienestar social de la región Piura. }\end{array}$} \\
\hline $\begin{array}{l}\text { Causas } \\
\text { directas }\end{array}$ & $\begin{array}{l}\text { De no aplicar principios de } \\
\text { ventaja competitiva y } \\
\text { estrategias sistémicas, no se } \\
\text { desarrollará el potencial } \\
\text { regional. }\end{array}$ & $\begin{array}{l}\text { Escasa igualdad de } \\
\text { oportunidades entre } \\
\text { la costa y sierra de la } \\
\text { región Piura. }\end{array}$ & $\begin{array}{c}\text { Demanda } \\
\text { insatisfecha de } \\
\text { insumos para la } \\
\text { producción de limón. }\end{array}$ \\
\hline & & \multirow{2}{*}{$\begin{array}{l}\text { Población débilmente } \\
\text { organizada, escasa } \\
\text { información, } \\
\text { necesidades básicas } \\
\text { insatisfechas. Pobre } \\
\text { diversidad económica } \\
\text { en agroindustria de } \\
\text { exportación y turismo. }\end{array}$} & \\
\hline $\begin{array}{l}\text { Causas } \\
\text { indirectas }\end{array}$ & $\begin{array}{l}\text { La falta de seguridad } \\
\text { jurídica, el mal desempeño } \\
\text { de las instituciones y las } \\
\text { barreras al desarrollo } \\
\text { comprometen la meta de } \\
\text { lograr competitividad. }\end{array}$ & & $\begin{array}{l}\text { Bajo contenido tecnológico, } \\
\text { escaso capital, bajos } \\
\text { niveles de producción y } \\
\text { productividad se traducen } \\
\text { en baja rentabilidad para } \\
\text { el productor. }\end{array}$ \\
\hline
\end{tabular}




\section{Anexo 2. Diagnóstico competitivo (FODA) de la cadena productiva}

\section{Fortalezas}

\section{Potencialidades}

- Uso doméstico, cosmético y propiedades medicinales del limón.

- Condición de fruto sin sustitutos directos en el consumo humano.

- Resistencia alta del limón fresco al transporte terrestre y marítimo (hasta 60 días) en frío (atmósfera controlada).

- Producto de alta productividad que responde a una inversión importante.

- Producción todo el año y en contraestación con México entre los meses de enero a marzo.

\section{Infraestructura}

- Alta disponibilidad de transporte marítimo, aéreo y terrestre.

\section{Desarrollo empresarial}

- Senasa es una entidad con mucha presencia en el campo.

- El limón no enfrenta problemas fitosanitarios que impidan su ingreso al mercado de Estados Unidos.

\section{Oportunidades}

\section{Nacional}

- Firma del tratado de libre comercio (TLC) con Estados Unidos.

- El índice de consumo nacional es muy bajo. México, 25 kg per cápita; Perú, 4 kg per cápita.

- Campaña de posicionamiento del pisco sour y el cebiche y mayores usos industriales.

- El limón no es hospedante de la mosca de la fruta.

\section{Externo}

- Ingreso formal a los mercados latinoamericanos: Bolivia, Ecuador, Colombia, Chile, Panamá, entre otros.

- Apertura del mercado americano.

- Alto porcentaje de la población de Estados Unidos son latinos; y de ellos, un buen número son mexicanos.

- Existe un mercado incipiente en la Unión Europea. 


\section{Debilidades}

\section{Potencialidades}

- Faltan normas de calidad (buenas prácticas agrícolas) para el mercado local.

- Falta de conocimiento en el manejo del cultivo y del riego.

- Inexistencia de un estándar de comercialización y embalaje.

- Faltan estudios de mercado para el consumo en fresco.

\section{Infraestructura y facilitación}

- Sistema de comercialización a través de mayoristas.

- Delicada situación financiera de los predios agrícolas.

- Insuficientes centros de acopio.

- Poco acceso a financiamiento.

- Limitaciones presupuestarias de Senasa.

- Ineficiencias en el funcionamiento de la Sunat.

\section{Desarrollo empresarial}

- Desorden en la comercialización en el mercado interno.

\section{Desarrollo exportador}

- Altos costos de embarque por puertos ineficientes.

\section{Sociales}

- Falta capacitar a los recursos humanos.

\section{Articulación empresarial}

- Existe una organización débil que requiere una mayor integración de los agricultores.

- Falta integración en la cadena industrial del limón.

\section{Amenazas}

\section{Nacional}

- Falta de planificación para la instalación de áreas limoneras, lo que puede disminuir el precio.

- Lluvias intensas y frecuentes, de acuerdo con los pronósticos de organismos especializados, que podrían afectar los cultivos (fenómeno de El Niño). 


\section{Anexo 3. Mapa de flujos económicos de la región Piura ${ }^{3}$}

Los principales ejes y corredores económicos en la región de Piura están asociados a:

1. El eje transversal del noreste: Piura-Sullana-Tambogrande-Las Lomas-Macará. Este eje denota un fuerte dinamismo derivado de la emergente actividad agroindustrial asociada al limón y al mango. Constituye, además, una ruta de comercio fronterizo de alto potencial en virtud del Tratado de Paz y Amistad con Ecuador.

2. El eje transversal del suroeste: Piura-Catacaos-La Arena-Vice-Sechura. Asociado a la producción agrícola pecuaria del valle del Bajo Piura, pero igualmente dinamizado por la actividad pesquera artesanal e industrial, así como por la actividad minera no metálica.

3. El eje transversal del noroeste: Piura-Paita y Piura-Sullana-Paita. Uno de los ejes más dinámicos como consecuencia de la intensa actividad turística, pesquera y portuaria de la zona de Paita. Amplias perspectivas para convertirse en la ruta hacia el puerto pivote o hub de Paita. Excelente ubicación geográfica para concentrar carga nacional e internacional.

4. El eje longitudinal del norte: Piura-Sullana-Talara-Máncora. Este eje se encuentra fuertemente dinamizado por actividades agrícolas, mineras (hidrocarbúricas), comerciales y turísticas; se trata de un eje de conexión interregional hacia la vecina región de Tumbes. Su dinámica económica es diversa y comprende, principalmente, la actividad agrícola del valle del Chira (algodón, arroz, maíz, sorgo granífero, frutales, hortalizas, cucurbitáceas), la relativamente pequeña actividad agroindustrial y agroexportadora, la actividad petrolera on-shore y of-shore en la cuenca de Talara, la pesca artesanal e industrial y la actividad turística en Los Órganos, Cabo Blanco y Máncora.

5. El eje andino del sureste: Piura-Buenos Aires-Carrasquillo-Canchaque-Huancabamba. Este eje sostiene la actividad agrícola, comercial y turística de la sierra huancabambina. Tiene un subeje variante de importancia estratégica que integra Huancabamba-SóndorTabaconas-Namballe-Eje Amazonas, importante alternativa y variante a la conexión Porculla -alta vulnerabilidad estacional en el previsto eje bioceánico-.

6. El eje andino central: asociado al tramo Piura-Morropón-Paltashaco-Pambarumbe-Chalaco, que integra a una de las zonas más deprimidas de la región. Zona de fuerte vocación agrícola de subsistencia, escasa conexión al mercado capitalista regional y alta vulnerabilidad ante la presencia de lluvias intensas asociadas al fenómeno de El Niño.

7. El eje norandino: Piura-Sullana-Ayabaca, variante del eje transversal noreste. Su destino es la fronteriza ciudad de Ayabaca, con fuerte tradición cultural, alta vocación agrícola y agroindustria artesanal. Este eje se caracteriza por presentar un alto grado de pobreza extrema, fuerte porcentaje de necesidades básicas insatisfechas, vocación hacia la agricultura de subsistencia, débil conexión a los mercados regional y nacional. Importante actividad turística pero muy estacional y concentrada. Poca o nula capacidad de explotación de sus riquezas arqueológicas y su legado cultural.

8. Otros corredores de integración intrarregional: asociados a las carreteras de penetración a la sierra, por ejemplo: Huancabamba-Socchabamba-Vadogrande, Sóndor-Huancabamba-Sapalache, Las Lomas-Sajinos, Sapalache-Ollería-El Molino, entre otros.

3. Fuente: Gobierno Regional de Piura. Plan de desarrollo regional concertado para el periodo 2003-2006. Piura, 2003. 